\title{
Medical Analytics for Healthcare Intelligence - Recent Advances and Future Directions
}

\author{
Tianhua Chen ${ }^{\mathrm{a}, *}$, Elpida Keravnou-Papailiou ${ }^{\mathrm{b}}$, Grigoris Antoniou ${ }^{\mathrm{a}}$ \\ ${ }^{a}$ Department of Computer Science, University of Huddersfield, Huddersfield, UK \\ ${ }^{b}$ Department of Computer Science, University of Cyprus, Cyprus
}

\section{Introduction}

Recent advances in information technology have facilitated the massive collection of big data in numerous areas, including the healthcare sector. Healthcare data exists in various forms that can be briefly grouped into two categories. First, there is clinical data that is directly related to patients and medical conditions. This data includes, but is not limited to, demographic data, patient history, lab test results, physical examinations, diagnostic analysis and medical notes. The proliferation of wearable devices also enables the collection of clinical data periodically through monitoring systems via wireless technology, which supports the real-time tracking of patient care and timely adjustment of treatment plans. A second category of healthcare data originates from the business side of healthcare, such as operational and equipment costs, and logistic and administrative data which may be utilised for the optimization of operational dynamics to support effective healthcare services and generally enhance utility in medical practice.

With massive data readily available, there is an ongoing paradigm shift in every corner of healthcare, since there are unprecedented opportunities to analyse and extract useful information from diverse, distributed and heterogeneous data sources to make more informed clinical decision making and enhance the

\footnotetext{
*Corresponding author

Email address: T.Chen@hud.ac.uk (Tianhua Chen)
} 
efficiency and performance of healthcare systems. Recent advances in machine learning and analytics can help reduce diagnostic and therapeutic errors that are inevitable in the human clinical practice, achieve consistency, improve the understanding of disease mechanisms and facilitate clinical decision support. It also transforms how medical research is conducted, and how healthcare is managed and delivered in a cost-effective manner.

In response to the great potentials of analytics in healthcare, there is an abundance of global investment and academic initiatives to promote innovative and transformative research in health informatics in recent years. To name a few, in August 2015, the British Engineering and Physical Sciences Research Council (EPSRC) invited applications for Programme Grant proposals that seek to address significant major research challenges that align to the Healthcare Technologies Grand Challenges, where the use of data analytics and intelligent solutions were explicitly emphasised in the grand challenges of Developing Future Therapies, Optimising Treatment and Transforming Community Health and Care. Horizon 2020, the biggest EU Research and Innovation programme, invested over 2 billion in better health for all during its first four years, encouraging the use of new technologies for data analytics and interpretation, such as artificial intelligence and computer modelling. In the U.S., the National Institutes of Health (NIH), the world's largest public funder of biomedical research, invests more than $\$ 32$ billion a year to enhance life and reduce illness and disability; a number of programs are directly related to medical analytics research, for example the Innovations in Biomedical Computational Science and Technology (R01) program, where support is offered for fundamental research in biomedical informatics and in the development of novel computational tools and technologies.

\section{Summary of Selected Papers}

To embrace the challenges and opportunities in designing and deploying intelligent healthcare systems, this special issue aimed to encourage submissions 
of scientific findings from both academia and healthcare industry that present the fundamental theory, techniques, applications and practical experiences in the context of designing, implementing or evaluating analytics for healthcare intelligence. Among the 121 manuscripts we've received, 72 were desk rejected for being out of scope in general, and the remainings were sent for peer review, each with at least two reviewers. Eventually 15 papers were accepted for publication in the special issue, giving an acceptance rate of just above $10 \%$. The accepted articles, summarised below, represent a broad spectrum of research for realizing the healthcare intelligence.

Time is of the essence in many clinical domains, necessitating its modelling in decision support systems for such domains. Four contributions of the Special Issue involve time considerations, namely the contributions by Bernardinia et al. [1], by Pokharel et al. 2], by Shifrin and Siegelmann [3] and by Bhatia et al. 4]. Bernardinia et al.'s work [1] deals with the domain of Type 2 Diabetes (T2D), where early prediction of a high risk for developing this life-threatening ailment is critically important. Their work is significant as it aims to improve current diagnostic practices that presently do not encompass the quantification of insulin resistance. Through the development of a predictive model based on a multiple instance learning boosting algorithm (MIL-Boost), they show that it is possible to accurately predict early the worsening of insulin resistance (low vs high T2D risk) in terms of the Triglyceride-glucose (TyG) index. As the authors show, the proposed MIL-based approach is able to extract hidden patterns from past electronic health record (EHR) temporal data, even not directly exploiting triglycerides and glucose measurements. A key advantage of their method lies in its ability to model the temporal evolution of longitudinal EHR data while dealing with small sample size and variability in the observations (e.g., a small variable number of prescriptions for non-hospitalized patients). The proposed algorithm could form the basic component of a clinical decision support system.

In working towards the management of diabetes, a personalised treatment scheme is proposed by Shifrin and Siegelmann [3] for patients with diabetes, which is based on a stochastic modelling of blood glucose level process and sta- 
tistical learning of possible reactions to various treatment actions. In particular, the reaction to insulin treatment is formulated as a Markov decision process, which is then learned by reinforcement learning. The generated personalised insulin care policy is different from the widely accepted modern approaches that generally rely on the constant equally dosed basal treatment combined with bolus injections at mealtime. The new method, though not necessarily medically intuitive, is able to prevent hypoglycemia, minimize high glucose duration and glycemic fluctuations, and automatically updates insulin management in response to personal or environmental change.

Diabetes is also addressed by Bhatia et al. 4, who propose a home-centric Urine-based Diabetes (UbD) monitoring system, which allows to track UbDrelated parameters in real-time using Internet of Things (IoT) sensors embedded across the toilet system. Temporal data can then be extracted which is further quantified into the Diabetes Infection Measure (DIM) over a fog computing platform. A Recurrent Neural Network is subsequently employed to predict the UbD infection based on DIM value and the corresponding temporal aspects, with results demonstrated through the self-organising mapping technique for enhanced visualization efficacy. Validated over real-world data of four individuals, the experimental results outperform conventional systems in terms of temporal delay, classification efficiency, prediction efficiency, reliability and stability analysis.

The need to compute similarities between patients using information from EHRs, thus finding similar cases for a target patient case, arises in the context of many clinical tasks. EHRs invariably include longitudinal data and hierarchically organized information. In their contribution, Pokharel et al. 2] present a novel approach for computing patient similarities from EHRs, whose central feature is a temporal tree representation; this is a temporal hierarchical representation based on temporal co-occurrence and preserves the compound information found at different levels in the data. The temporal tree representation is augmented using the doc2vec embedding technique. Through their proposal they aim to address key challenges associated with EHR data, such as temporal 
aspects, multivariate, heterogeneous and irregular data, as well as data sparsity. The viability of their approach is empirically demonstrated, along with several state-of-the-art benchmarks, on a dataset of real world ICU EHRs, for the task of identifying patients with a specific target diagnosis.

Radiology images are central to medical data. As Monshi et al. [5] report in their survey type contribution, substantial progress has been made towards implementing automated radiology reporting models based on deep learning (DL), where the generation of radiology coherent paragraphs that do more than traditional medical image annotation, or single sentence-based description, has been the subject of recent academic attention. This is a challenging, multidisciplinary application-based endeavor as it brings together neural networks, deep learning and natural language processing with the aim of bridging visual medical features and radiologist text. The authors' expectation is that this area of research will grow in the near future. The presented survey, that constitutes a noteworthy addition to the literature for researchers interested in DL, particularly those interested in applying DL to radiology reporting, focuses on the following critical challenges: understanding radiology text/image structures and datasets, applying DL algorithms (mainly CNN and RNN), generating radiology text, and improving existing DL based models and evaluation metrics. A critical discussion with future research recommendations gives the authors' vision of the progress to come in this particular field of medical analytics.

Knowledge discovery from omics data has become a common goal of current approaches to personalised cancer medicine and understanding cancer genotype and phenotype. With omics data characterised by high dimensionality and relatively small sample sizes with small signal-to-noise ratios, Alzubaidi et al. [6] propose a deep feature learning model based on a set of non-linear sparse Auto-Encoders that are deliberately constructed in an under-complete manner to detect a small proportion of molecules that can recover a large proportion of variations underlying the data This is followed by the introduction of a novel weight interpretation technique that helps to deconstruct the internal state of such deep learning models to reveal key determinants underlying its latent rep- 
resentations. Experiments reveal that the discovered biomarkers demonstrate computational and biological relevance, as well as the capability to construct highly accurate and reliable prediction models.

Early and accurate diagnosing Kawasaki Disease (KD), which is a leading cause of acquired heart disease in children, is impracticable for its unknown pathogenesis and the lack of pathognomonic features. Wang et al. [7] exploit incomplete and multi-source clinical data based on a cohort of 10,367 patients for the early assessment of KD. To address the data incompleteness that leads to group-based missing patterns associated with various clinical measures, the multi-source structure of clinical data is perceived using a matrix-based representation. A convolutional neural network is then employed to extract features and subsequently optimised to work with the multiway data, achieving superior performance in comparison to popular benchmarks.

Self-monitoring and behavioral modification are key to the successful management of chronic health conditions. Dragoni et al. 8 present an AI system that receives input regarding a person's food intake and, where unhealthy activity is detected, shows the user a natural language message explaining the wrong behavior and its consequences. The system applies symbolic AI techniques (ontologies and logical reasoning) to detect situations where personal behavior deviates from desirable patterns. In such cases, the system applies persuasive explanation and natural language generation techniques to generate and deliver a message to the user. The evaluation included validation of the AI system's outputs by domain experts; feedback on usability by users; and crucially, demonstration of reduced negative behavior compared to a control group without AI intervention.

Assessment of motor performance of patients after a stroke is important in determining proper therapy. At present, this assessment is carried out by clinicians based on a number of human-administered clinical scales. This approach depends on the clinician's experience, and there is an element of variation of assessment. Zhou et al. 9] propose an assessment framework for persons after stroke via surface electromyography, based on four types of selected arm move- 
ments, with the aim of establishing a quantitative measure of stroke severity. The linear discriminant analysis (LDA), random forest algorithm (RF) and support vector machine (SVM) are adopted, trained and used for stroke patients quantitative recognition. Results are promising and suggest that the, currently predominant, qualitative assessment by experts can be complemented by quantitative assessment methods.

Optical Coherence Tomography (OCT) is a rapidly developing non-invasive 3D imaging approach, which has been widely used in the examination of eye diseases. Owing to the potential speckle noise that may inherit from image acquisition process and thus obscure the anatomical structure, Yan et al. 10] propose a novel approach that utilises a multi-frame fusion mechanism by merging multiple scans for the same scene and then recovers missing signals in one pixel through movements of sub-pixels. Evaluated on 20 OCT volumes with 5120 images, the experimental results on multiple assessment metrics confirmed the effectiveness of the proposed method. This is followed by the evaluation of the speckle reduced images on the detection of the retinal layer boundaries acquired through deep neural networks, which improves the precision of the layer segmentation in comparison with state-of-the-art medical image enhancement methods.

While working with data in the form of functional Magnetic Resonance Imaging (fMRI), which is a non-invasive imaging technique for measuring brain activity, few analytical approaches simultaneously take into account the multivariate aspect and the connectivity features of the brain. Brahim and Farrugia [11] propose a novel multimodal analysis approach, which combines features extracted through Graph Fourier Transform (GFT) on a structural graph and several statistical metrics of resting state fMRI (rs-fMRI) time series defined on the same brain regions as the structural graph. Evaluated on the popular Autism Brain Imaging Data Exchange data set, the proposed approach demonstrates its superiority and efficiency, which beats those using functional connectomes or complex functional network measures as features.

Osteomyelitis (OM) is a serious bacterial infection in children requiring ur- 
gent antibiotic therapy. Wu et al. [12] develop a Bayesian Network (BN) model to guide individually targeted antibiotic therapy at point-of-care, by predicting the most likely causative pathogen in children with $\mathrm{OM}$ and the antibiotic with optimal expected utility. The BN, which integrates data with critical expert knowledge under a causal inference framework, explicitly models the complex relationship between the unobserved infecting pathogen, observed culture results, and clinical and demographic variables. Experimental results demonstrate the strong agreement of antibiotic choices made by the proposed model and those of clinical experts, with BN-recommended antibiotics rated optimal or adequate by experts in $82-98 \%$ of 81 cases sampled from the tested cohort.

The segmentation of brain Magnetic Resonance (MR) images facilitate the identification of homogeneous artifacts along with edges and textures. However, segmentation is typically complicated due to their inherent ambiguous structures and inhomogeneities in grayscale intensities. Singh [13] proposes an adaptive image segmentation method, whereby the neutrosophic set theory and neutrosophic entropy information (NEI) are used to measure uncertainties in MR images in terms of the neutosophic information such that the gray level at the location of maximum NEI value can be selected as the segmentation threshold. Validated through the segmentation of Parkinson's disease MR images, the proposed method outperforms a number of existing well-known image segmentation approaches with fewer computational overheads.

Sharing medical data across institutions is a common practice in collaborative clinical research that may involve complicated privacy preserving issues. Li et al. 14 propose a multicenter Random Forest (RF) prognosis prediction model that enables federated clinical data mining from horizontally partitioned datasets. This is supported with a novel data enhancement approach based on a differentially private generative adversarial network customized to clinical prognosis data, as well as an importance ranking step designed for feature selection without sharing patient-level information. Evaluated on colorectal cancer data from the US and China, experimental results demonstrate that the proposed multicenter RF can provide better prediction performance in terms of 
discrimination and calibration ability than the centrally trained RF model and popular centrally trained machine learning methods while following the privacypreserving rules in both groups.

Last but not least, Xiang et al. [15] presents a status analysis on the investigation of differences in the attitudes and perceptions regarding the implementation of medical AI between healthcare workers and non-healthcare workers. An online questionnaire was designed to investigate the perceptions, receptivity and demands of general public on AI in medicine between October 13 and October 30,2018 , with a total number of 2,780 participants from 22 provinces in China enrolled, among which healthcare workers accounted for $54.3 \%$. Through descriptive statistics, high levels of receptivity (nearly 100\%), demands (approximately $80 \%$ ), and expectations (100\%) were expressed among different age groups. There was a very large gap between current availability of and public demands for intelligence services, with over $90 \%$ of healthcare workers having expressed a willingness to devote time to learning about AI and participating in AI research. While there is a strong demand for intelligent assistance in numerous areas, including imaging and pathology departments, outpatient services and surgery, such investigation of public opinions over the popularity and expectations of healthcare intelligence in practice could potentially inform the research direction and relevant resource allocation to the further advancement of medical analytics and artificial intelligence in medicine.

\section{Future Directions}

Explainability, i.e. the ability to present and adequately justify the rationale behind decisions or recommendations, has always been a highly sought requirement for $\mathrm{AI}$ systems in the healthcare domain. Why are explanations important if a system can achieve high performance? The reason is simple and obvious: no medic or healthcare provider, who would ultimately be responsible for their actions towards patients, could take at face value the outcome of a computer-based system, no matter how much knowledge, expertise or intelli- 
gence is encoded in it. Explainability has an important added value too, as it can render AI systems able cognitive tutors to medical students, over and above to being competent problem solvers. Even in the early days of knowledge-based systems, explanations could range in type and form, from simple canned text to inference rule chains, to deeper causal explanations or meta-level strategic explanations, that could be dynamically adapted - in detail and concept significance - to the needs of different users. The technical challenges associated with the provision of explainable AI systems, in particular for medical domains, were not insignificant as it soon transpired that explanations impinge on the architectural backbone of these systems. Simply said, adequate and convincing explanations cannot be just added to an existing system, but should be designed as an intrinsic architectural feature from the start. The shift from knowledge-based to data-driven AI systems in medicine, that started two decades ago, has been getting stronger through the ever expanding availability of data coupled with the development of medical analytics for healthcare intelligence and high performances infrastructure. For a period of time, these developments overshadowed the importance of explainability. However, the need for transparency and interpretability in connection with the so called "black-box" approaches is recently coming more and more prominently into the AI arena. The re-acknowledgement of the importance of explainability is clearly evident through the emerging field of "Explainable AI" which is bringing back the previous challenges of explanation provision, but now rooted on data rather than knowledge. Although there could be exceptions, such as image or signal processing applications where high performance could be an adequate qualifier for entrance into the healthcare domain, generally-speaking the nature of healthcare, human life and well-being, may render black-box approaches untenable no matter how high their performance is. Explainability can unlock their potential as important healthcare tools by rendering them transparently interpretable.

Undoubtedly, data and its harnessing through AI-based analytic methods with the objective of creating important tools, models and applications for the benefit of society is here to stay and grow stronger. The previous key words 
"Knowledge Society" and "Knowledge Economy" have now been rightly replaced by the terms "Data Society" and "Data Economy" to emphasize the central role that data has now acquired. However, the fact that data is currently readily available and often in large sizes, does not necessarily mean that data is a panacea. Putting aside the technical challenges associated with data management and processing, data could also hide biases which if not detected and addressed by appropriate analytic methods would simply result in such biases being passed on and perpetuated by the generated models. Invariably, medical practice in some specific domains, as well as attitudes in other sectors of human life, could be associated with certain biases against groups of society such as minority groups, gender groups, to the extent that it would not be a misnomer to refer to such practices as neither fully ethical nor socially inclusive. If medical analytics for healthcare intelligence is to serve the good of society at large, in an ethically accepted and inclusive manner, it should be in a position to unravel potential biases in the data and to ensure the inclusiveness and validity of the adopted data. This way, the new analytic models thus induced will surpass the limitations and biases of human practices, otherwise they will simply reproduce them. Rendering such models transparent and explainable can be catalytic in pruning a medical data bank from biases, misconceptions or even unethical practices.

A significant body of recent advances focuses on proposing models of high performance or demonstrating their competence over medical experts. However, there are still numerous gaps on integrating these capabilities into clinical practice, especially due to a lack of sufficient consideration in human machine interaction. Challenges in this area lie in various facets, ranging all the way from the cognitive willingness of attempting an AI system to integrating it seamlessly with medical practice. With clinicians likely to expect working with AI systems in the same way they work with fellow colleagues, having a friendly interface with associated performance summary statistics is far from enough to deploy AI systems into practical use. A lot more functionality is actually required, such as the demonstration of model expertise, bias and limi- 
tations; the communication of confidence levels and inferences behind decisions delivered through an interpretable and transparent form; and even discource functionality enabling humans and AI to debate approaches and results. Another barrier that hinders the human AI interaction is the de-skilling of medical personnel, who have to constantly keep up to date with, and adjust to the rapid evolution of AI and medical knowledge and technology, which is somehow in conflict with already overwhelmed workload, with some even fearing that they may eventually be replaced by AI.

The enhancement of human AI interaction brings confidence and trust of healthcare professions in AI technology, which is vital to the adoption of AI systems. However, it must be recognised that the public acceptance is not yet high from numerous surveys, with major issues in areas such as data sharing and regulation that should be continuously improved upon to boost public trust. The hesitation towards data sharing lies in a lack of public trust on how the data is handled, stored and utilised, especially for purposes beyond their direct care; often the cause is poor communication between staff and patients on the benefits of data sharing, with staff members sometimes even unclear over their legal responsibilities, hence leading to poor engagement. This calls for the creation of a secure and transparent environment with clarity and visibility for the well-functioning of data-sharing ecosystem. However, it is inspiring to see advances made in recent years at the national scale, such as the approval of a cloud-based online medical imaging platform that assists the diagnosis of heart conditions by the US Food and Drugs Administration (FDA). With considerations in algorithms, models, training and selected features to deploy machine learning systems of analysing medical imaging, the FDA has assembled teams to oversee and anticipate future developments in AI-driven medical software. Although the AI algorithms currently play more the role of decision support tools, which aim to provide advice instead of making clinical decision replacing doctors, the public trust, safety and ethics will be an urgent concern to healthcare regulators for widespread deployment of AI in medical practice.

Data analytics is usually associated with learning from big data, and has 
broadly been the main effort of machine learning research and practice in the past years. Recent advances in healthcare, for example using AI algorithms to interpret digital scans or brain images, rely on such methods. However, there are certain applications where learning from small data is essential. A first area of application in healthcare is the desire to derive locally adapted models. For example, one may wish to develop a risk assessment model for suicide tailored to a particular area to get better results: a local model will adapt better to the specific characteristics of the local population, and may consider local factors, such as referring and treating hospitals, as placeholders for underlying socioeconomic conditions that are not otherwise represented in the healthcare data. Secondly, it is not uncommon for biological datasets to contain fewer than 100 samples: as of March 2020, 74.6\% of the 4348 curated datasets provided by Gene Expression Omnibus count 20 or fewer samples. This is typical in rare cancers and diseases, in experimental therapy treatments, and whenever the measurement costs are high. And thirdly, if we think of the Covid19 pandemic that is ravaging the world at the time of writing, waiting until a sufficient data basis is established for big data approaches to work is not an option. Instead, it is essential to learn from the little data available, rerunning algorithms in regular intervals to reflect the increasing data basis and possible data drift. Such an approach to emerging pandemics enables a more accurate response on the basis of the limited data available at the time, but evolving as more data comes in. This approach also relies on the possibility of carrying out comprehensive data analyses with as little effort and time as possible; this relates to the emerging AutoML (automated machine learning) trend that seeks to add automation to the data analysis process. From a technical perspective, smallsample data present particular statistical estimation challenges, as overfitting, overestimating and model selection difficulties are exacerbated.

As we stated earlier, healthcare intelligence has come to rely mostly on the analysis of data through statistics and machine learning. However, we believe that it is important to further explore the combination of knowledge-based

AI and machine learning. The biomedical domain is described by a number 
of relevant ontologies, and these resources, reflecting decades of medical knowledge, can play a crucial part in data analysis. For example, existing ontologies can be used a posteriori to provide explanations for insights derived through deep learning - we have discussed the importance of explainability earlier. Alternatively, existing ontologies may be used a priori to define parts of the layers and nodes in a deep learning network. In addition, ontologies and knowledge can play an important role in AutoML as well. They can be used to represent knowledge contained in the machine learning literature, as well as knowledge derived by experiences of senior data analysts, regarding the best way to design data analysis pipelines depending on (a) the application domain at hand, (b) the type of data analysis problem, (c) the profile of available data and (d) preferences of users, e.g. in terms of explainability or speed of analysis. We expect that such knowledge will be at the heart of the most successful AutoML products for the healthcare domain.

\section{Acknowledgments}

We would like to thank Professor Carlo Combi for kindly providing the chance to organise this special issue. We would also like to thank all the authors who have contributed to this special issue. Special thanks go to the many reviewers as well, for their efforts to guarantee the high quality of this special issue.

\section{References}

[1] M. Bernardini, M. Morettini, L. Romeo, E. Frontoni, L. Burattini, Early temporal prediction of type 2 diabetes risk condition from a general practitioner electronic health record: A multiple instance boosting approach, Artificial Intelligence in Medicine (2020) 101847.

[2] S. Pokharel, G. Zuccon, X. Li, C. P. Utomo, Y. Li, Temporal tree representation for similarity computation between medical patients, Artificial Intelligence in Medicine (2020) 101900. 
[3] M. Shifrin, H. Siegelmann, Near-optimal insulin treatment for diabetes patients: A machine learning approach, Artificial Intelligence in Medicine (2020) 101917.

[4] M. Bhatia, S. Kaur, S. K. Sood, V. Behal, Internet of things-inspired healthcare system for urine-based diabetes prediction, Artificial Intelligence in Medicine (2020) 101913.

[5] M. M. A. Monshi, J. Poon, V. Chung, Deep learning in generating radiology reports: A survey, Artificial Intelligence in Medicine (2020) 101878.

[6] A. Alzubaidi, J. Tepper, A. Lotfi, A novel deep mining model for effective knowledge discovery from omics data, Artificial Intelligence in Medicine $104(2020) 101821$.

[7] H. Wang, X. Tan, Z. Huang, B. Pan, J. Tian, Mining incomplete clinical data for the early assessment of kawasaki disease based on feature clustering and convolutional neural networks, Artificial Intelligence in Medicine (2020) 101859 .

[8] M. Dragoni, I. Donadello, C. Eccher, Explainable ai meets persuasiveness: Translating reasoning results into behavioral change advice, Artificial Intelligence in Medicine (2020) 101840.

[9] Y. Zhou, J. Zeng, H. Jiang, Y. Li, J. Jia, H. Liu, Upper-limb functional assessment after stroke using mirror contraction: A pilot study, Artificial Intelligence in Medicine (2020) 101877.

[10] Q. Yan, B. Chen, Y. Hu, J. Cheng, Y. Gong, J. Yang, J. Liu, Y. Zhao, Speckle reduction of oct via super resolution reconstruction and its application on retinal layer segmentation, Artificial Intelligence in Medicine (2020) 101871.

[11] A. Brahim, N. Farrugia, Graph fourier transform of fmri temporal signals based on an averaged structural connectome for the classification of neuroimaging, Artificial Intelligence in Medicine (2020) 101870. 
[12] Y. Wu, C. McLeod, C. Blyth, A. Bowen, A. Martin, A. Nicholson, S. Mascaro, T. Snelling, Predicting the causative pathogen among children with osteomyelitis using bayesian networks-improving antibiotic selection in clinical practice, Artificial Intelligence in Medicine (2020) 101895.

[13] P. Singh, A neutrosophic-entropy based adaptive thresholding segmentation algorithm: A special application in mr images of parkinson's disease, Artificial Intelligence in Medicine (2020) 101838.

[14] J. Li, Y. Tian, Y. Zhu, T. Zhou, J. Li, K. Ding, J. Li, A multicenter random forest model for effective prognosis prediction in collaborative clinical research network, Artificial Intelligence in Medicine 103 (2020) 101814.

[15] Y. Xiang, L. Zhao, Z. Liu, X. Wu, J. Chen, E. Long, D. Lin, Y. Zhu, C. Chen, Z. Lin, et al., Implementation of artificial intelligence in medicine: Status analysis and development suggestions, Artificial Intelligence in Medicine 102 (2020) 101780. 